\title{
LONGITUD CERVICAL Y FIBRONECTINA EN EL SÍNTOMA DE PARTO PREMATURO *
}

\author{
Carlos Treuer P. ' , Víctor Quiroz G. ${ }^{1}$, Jorge Cabrera D. ${ }^{2}$, Carmen Soto L. ${ }^{2 a}$, \\ Heriberto Araneda C. $P h D^{2 b}$ \\ ${ }_{1}^{1}$ Servicio de Obstetricia y Ginecología, Hospital Guillermo Grant B. \\ 2 Departamento de Obstetricia y Ginecología, Facultad de Medicina, Universidad de Concepción.
}

a Matrona. ${ }^{\mathrm{b}}$ Tecnólogo médico.

* Trabajo financiado por Proyecto DIUC No 201.084.002-1.0

\section{RESUMEN}

Antecedentes: En Chile el parto prematuro es la principal causa de morbilidad y mortalidad perinatal. Objetivo: Evaluar la utilidad clínica de la longitud cervical y la detección de fibronectina, en la predicción de parto prematuro. Método: Estudio observacional prospectivo, de corte transversal en 86 pacientes que consultaron por síntomas de parto prematuro (SPP), con membranas indemnes y edad gestacional entre las 22 y 34 semanas. El grupo estudio formado por gestantes con parto prematuro dentro de los 7 días. Al ingreso se tomó muestra cervical para fibronectina y se midió longitud cervical mediante ultrasonografía transvaginal. Resultados: De las 86 gestantes estudiadas, en $13(15 \%)$ se produjo el parto dentro de los 7 días. La curva ROC mostró un área bajo la curva para longitud cervical de 0,475 y sensibilidad $31 \%$ (4/13), especificidad $96 \%$ (70/73), valor predictivo positivo $57 \%$ (4/7) y valor predictivo negativo $89 \%(70 / 79)$, riesgo relativo positivo 5,0 y riesgo relativo negativo $0,47(p=0,014)$. El área bajo la curva para fibronectina fue 0,92 con una sensibilidad $77 \%$ (10/13), especificidad $90 \%$ (66/73), valor predictivo positivo (10/17) $59 \%$ y valor predictivo negativo $96 \%(66 / 69)$, riesgo relativo positivo 13,48 y riesgo relativo negativo $0,43(p<0,001)$. Conclusión: En pacientes con síntoma de parto prematuro, cuello no remodelado y fibronectina cervical negativa, se asocian con bajo riesgo de parto prematuro. La fibronectina positiva, fue mejor predictor de parto prematuro dentro de los siete días y la longitud cervical fue superior para descartarlo.

\section{PALABRAS CLAVE: Parto prematuro, fibronectina, cuello uterino, ultrasonografía transvaginal}

\section{SUMMARY}

Background: In Chile the preterm labor is the main cause of perinatal morbidity and mortality. Objective: To evaluate the clinical utility of the cervical length and fibronectin determination in predicting preterm delivery. Method: A prospective observational and transactional study was made, in 86 patients with preterm labor symptoms and gestational age between 22 and 34 weeks. The study group included patients with labor and delivery within a week of admission. From each patient a cervico-vaginal specimen was obtained to measure fibronectin and transvaginal ultrasonographic evaluation of the uterine cervix. Results: From 86 patients admitted to the study, thirteen had preterm labor (15\%). The ROC curve showed an area under curve for cervical length of 0.475 and sensitivity $31 \%$ (4/13), specificity $96 \%(70 / 73)$, positive predictive value $57 \%(4 / 13)$, negative predictive value $88 \%(70 / 79)$, positive relative risk was 5.0 , and the negative relative risk 0.47 ( $p=0.014)$; for fibronectin was 0.92 and sensitivity $77 \%(10 / 13)$, specificity $90 \%(66 / 73)$, positive predictive value $59 \%(10 / 17)$, negative predictive value $96 \%(66 / 69)$, positive relative risk of 13.48 , negative relative risk $0.43(p=0.014)$. Conclusion: In patients with premature labor symptoms, the cervical lengths 
none shortened and negative fibronectin result were associated with a low risk of having a preterm labor. Positive fibronectin was a better predictor of premature birth in a week of admission and the cervical length was the best in ruling out the occurrence of a premature birth.

\section{KEY WORDS: Preterm labor, fetal fibronectin, uterine cervix, transvaginal ultrasonography}

\section{INTRODUCCIÓN}

El parto prematuro (PP) es una condición patológica que tiene múltiples etiologías, estos procesos comparten una vía terminal común caracterizada por cambios en la matriz extracelular y disrupción de la interface coriónica decidual que llevan a contracciones miometriales y remodelación cervical $(1,2)$. El parto prematuro espontáneo antes de las 37 semanas ocurre entre el 7 al $10 \%$ de las gestantes y entre el 3 y $4 \%$ antes de las 34 semanas (3). Los métodos usados tradicionalmente para la identificación de pacientes con síntomas de PP incluyen: examen físico, historia obstétrica, signos premonitorios y factores sociodemográficos de riesgo que son poco sensibles y específicos (4). Actualmente a pesar del uso de tocolíticos, la tasa de prematurez en el mundo se ha mantenido estable en la últimas décadas $(5,6)$.

El mayor desafío que enfrenta la obstetricia, es avanzar en la etiopatogenia del PP, para así desarrollar estrategias de manejo preventivas (7). Nuestra limitada comprensión de los mecanismos etiopatogénicos, nos llevó a evaluar marcadores de la vía terminal común, para reducir la hospitalización e intervenciones innecesarias $(8,9)$. Las fibronectinas son una familia de proteínas multifuncionales del plasma y la matriz extracelular involucradas en la adhesión celular, opsonización y remodelación tisular. El parto prematuro se acompaña de mayor actividad proteolítica coriónica-decidual y liberación de componentes específicos de la matriz extracelular coriónica en las secreciones cérvicovaginales (10). La medición de la longitud cervical con ultrasonido hoy en día es aceptada como un procedimiento rápido y objetivo para la identificación de pacientes con modificaciones cervicales y en riesgo de parto prematuro, y tiene la ventaja que es un procedimiento fácil y accesible pero operador y equipo dependiente.

El objetivo de este estudio fue evaluar la utilidad clínica de la longitud cervical y de la fibronectina en el síntoma de parto prematuro.

\section{MATERIAL Y MÉTODO}

El estudio se realizó en el Servicio de Obstetricia y Ginecología del Hospital Guillermo Grant Be- navente de Concepción, entre noviembre de 2002 a octubre de 2004. Se hizo un estudio observacional, prospectivo de corte transversal, que incluyo a 86 pacientes con edad gestacional entre las 20 y 34 semanas que consultaron por síntomas de parto prematuro con membranas indemnes. El protocolo del estudio fue aprobado por el comité de ética de la Dirección de Investigación de la Facultad. Al momento de la consulta, y previo consentimiento informado, de cada paciente se obtuvo una muestra del orificio cervical externo, con tórula de Dacron que se dejó in situ por 30 segundos, luego se homogenizó en buffer y se procesó dentro de una hora, almacenada a $-20 \stackrel{\circ}{\circ}$ hasta el momento del ensayo. La determinación de fibronectina se hizo mediante reactivo de Adeza Biomedical Corporation, Sunnyvale, $\mathrm{Ca}$. USA. La evaluación ultrasonográfica transvaginal, se realizó en un equipo Toshiba modelo ECCO CEA. El valor de corte para la longitud cervical fue de $\leq 25 \mathrm{~mm}$ y para fibronectina $\geq 50 \mathrm{ng} / \mathrm{ml}$.

El grupo en estudio lo conformaron gestantes cuyo intervalo entre el parto y el ingreso fue menor o igual a 7 días. El manejo clínico de las pacientes se realizó según las normas del Servicio y los resultados obtenidos no estuvieron disponibles para los residentes. Se consideró criterio de exclusión a las siguientes patologías del embarazo: colestasia intrahepática, preeclampsia, diabetes gestacional, diabetes mellitus, infección urinaria, ruptura prematura de membranas, isoinmunización $R h$ "D", pacientes en tratamiento médico de patologías crónicas, embarazo múltiple y relación sexual 24 horas previas a la toma de muestra.

Los resultados fueron objeto de análisis estadístico con test no paramétricos, incluyendo curva ROC. Un valor $p<0,05$ fue considerado significativo. Los análisis estadísticos se hicieron con el programa SPSS 12.0 para Windows, Chicago, IL. USA.

\section{RESULTADOS}

La prevalencia de parto prematuro en la muestra fue de $15 \%(13 / 86)$, y no hubo diferencia significativa en las variables demográficas (Tabla I). El análisis de la curva ROC (Figura 1), para resultados de fibronectina y acortamiento cervical mostró que el área bajo la curva para los niveles de fibronectina cervical $(0,92)$ fue significativamente mayor 
que el área para el acortamiento cervical $(0,48)$. La longitud cervical presentó una sensibilidad de $31 \%$ (4/13), especificidad de $96 \%$ (70/73), valor predictivo positivo de $31 \%(4 / 13)$ y valor predictivo negativo de $88 \%$ (70/79), riesgo relativo positivo de 5,0 y riesgo relativo negativo de $0,47\left(x^{2}: 5,13\right.$; $\mathrm{p}=0,014)$. La fibronectina mostró una sensibilidad de $77 \%$ (10/13), especificidad de $90 \%$ (66/73), valor predictivo positivo de $59 \%$ (10/17), valor predictivo negativo de $96 \%$ (66/69), riesgo relativo positivo de 13,48 y riesgo relativo negativo de $0,43\left(x^{2}: 32,44 ; p\right.$ $<0,001$ ) (Tabla II).

\section{DISCUSIÓN}

El objetivo de este trabajo fue evaluar la utilidad clínica de la longitud cervical y la fibronectina, en gestantes con síntomas de parto prematuro. Las estrategias para una detección precoz de PP basados en factores de riesgo no han sido efectivas por lo que se ha buscado otras alternativas. Aparte de identificar a las pacientes en riesgo de tener un $\mathrm{PP}$, también es importante poder saber las que no estarán expuestas al riesgo, para no someterlas a hospitalización y tratamientos innecesarios. No se consideró en esta investigación los PP causados

Tabla I

\section{CARACTERÍSTICAS DE LAS PACIENTES EN ESTUDIO}

\begin{tabular}{lccc}
\hline $\begin{array}{l}\text { Características } \\
\text { maternas }\end{array}$ & SPP & PP & Valor p \\
\hline Edad materna (años) & $25(14-39)^{\star}$ & $24(15-38)^{\star}$ & 0,720 \\
Menores de 18 años & $9 / 73(12 \%)$ & $3 / 13(23 \%)$ & 0,078 \\
Solteras & $53 / 73(72 \%)$ & $9 / 13(69 \%)$ & 0,135 \\
Escolaridad (años) & $8(0-14)^{\star}$ & $8(2-12)^{\star}$ & 0,802 \\
Edad gestacional & & & \\
(semanas) & $30(25-35)^{\star}$ & $30(21-34)^{\star}$ & 0,791 \\
$\begin{array}{l}\text { Antecedentes de } \\
\text { aborto }\end{array}$ & $14 / 73(19 \%)$ & $3 / 13(23 \%)$ & 0,089 \\
Nulíparas & $31 / 73(42 \%)$ & $7 / 13(53 \%)$ & 0,068 \\
Cerclaje & $2 / 73(2,7 \%)$ & $1 / 13(7,6 \%)$ & 0,073 \\
\hline
\end{tabular}

SPP: Síntomas de parto prematuro.

PP: Parto prematuro. *Mediana (rango)

por RPM o los producidos por causa médica. En nuestro estudio al igual que en la literatura, se observa un mayor porcentaje de PP en mujeres con nivel socioeconómico bajo y edades menores de 18 años (4). La manera ideal de confirmar o descartar un parto prematuro no existe. El uso de la fibronectina en esta patología tiene el inconveniente de su costo, pero la ventaja demostrada en nuestro

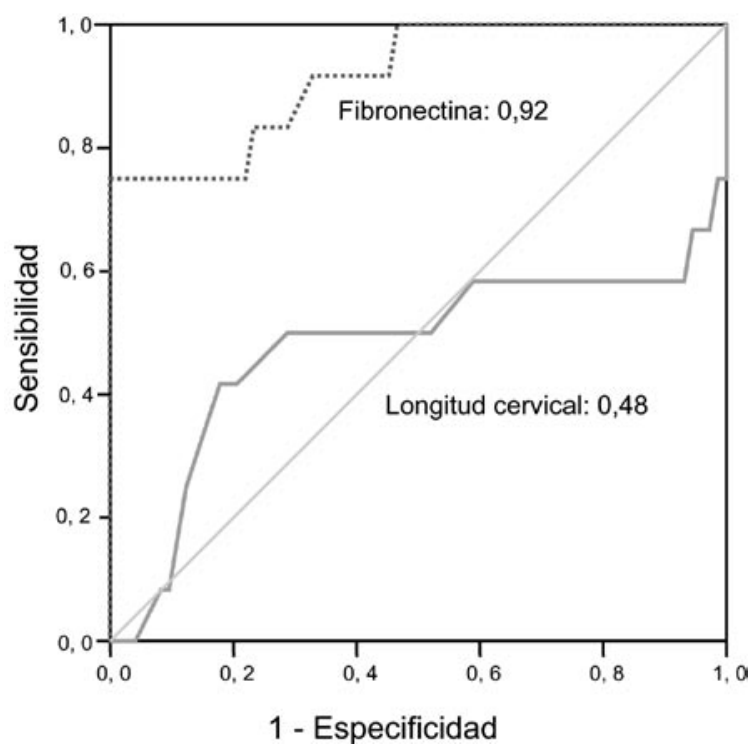

Figura 1. Análisis de curva ROC muestra que área bajo la curva para los niveles de fibronectina cervical $(0,92)$ fue significativamente mayor que el área para el acortamiento cervical $(0,48)$.

\section{Tabla II}

EFICACIA DE LA DETERMINACIÓN DE FIBRONECTINA Y LONGITUD CERVICAL PARA LA PREDICCIÓN DE PARTO PREMATURO

\begin{tabular}{lcccccc}
\hline \multicolumn{8}{c}{ PARTO PREMATURO } \\
& $\begin{array}{c}\mathrm{S} \\
(\%)\end{array}$ & $\begin{array}{c}\mathrm{E} \\
(\%)\end{array}$ & $\begin{array}{c}\text { VPP } \\
(\%)\end{array}$ & $\begin{array}{c}\text { VPN } \\
(\%)\end{array}$ & $\mathrm{RR}+\mathrm{RR}-$ \\
\hline${ }^{*}$ Fibronectina \\
$\begin{array}{l}\text { cervical } \\
50 \mathrm{ng} / \mathrm{ml} \\
{ }^{*} \text { Longitud } \\
\text { cervical } \\
<25 \mathrm{~mm}\end{array}$ & 77 & 90 & 59 & 96 & 13,5 & 0,4 \\
\hline
\end{tabular}

S:sensibilidad. E: especificidad. VPP: valor predictivo positivo. VPN: valor predictivo negativo. RR: riesgo relativo. $\left.\left.{ }^{*} x^{2}=32,44 ; p<0,001\right) ;{ }^{* *} x^{2}=5,13 ; p=0,014\right)$.

estudio radica en su alto valor predictivo negativo (96\%), lo que concuerda con varios autores (5-7). También cuando no estaba presente, el riesgo relativo negativo de tener un PP era igual a la población general.

La medición de la longitud cervical tiene la ventaja de su bajo costo si se tiene el equipamiento necesario y la oportunidad de tener un resultado rápido. Cuando la longitud cervical es mayor a $25 \mathrm{~mm}$ el riesgo relativo negativo es igual a la población 
general. Al igual que en la literatura este estudio demostró que el valor de la fibronectina fetal y la medición de la longitud cervical esta en descartar el PP, más que en confirmarlo (9), por lo que creemos sería útil para el manejo de estas pacientes adicionar a la medición de la longitud cervical el test rápido de fibronectina (11-12). La conquista médica de esta patología no se ve cercana, aún falta mucha investigación para conocer las determinantes etiopatogénicas.

\section{CONCLUSIÓN}

En gestantes con síntoma de parto prematuro, un cuello uterino no acortado y fibronectina negativa, se asociaron con bajo riesgo de parto prematuro. La fibronectina positiva, fue mejor predictor de parto prematuro dentro de siete días, y la longitud cervical fue superior para descartarlo.

\section{BIBLIOGRAFÍA}

1. Schmitz T, Maillard F, Bessard-Bacquaert S, Kayes G, Fulla Y, Carbol D, Goffinet F. Selective use of fetal fibronectin detection after cervical length measurement to predict spontaneous preterm delivery in women with preterm labor. Am J Obstet Gynecol 2006;194(1):13843.

2. lams GD, Goldenberg RL, Mercer BM, Moawad AH, Meis PJ, Das AF, et al. The Preterm Prediction Study: can low-risk women destined for spontaneous preterm birth be identified? Am J Obstet Gynecol 2001; 184:652-5.

3. Honest H, Bachmann LM, Gupta JK, Kleijnen J, Khan KS. Accuracy of cervicovaginal fetal fibronectin test in predicting risk of spontaneous preterm birth: systematic review. BMJ 2002;325(7359):301. Hallado en: http://www.pubmedcentral.nih.gov/picrender.fcgi?artid $=117763 \&$ blobtype $=$ pdf.

4. Lockwood CJ, Kuezynski E. Markers of risk for preterm delivery. J Perinat Med 1999;27:5-20.

5. Ingemarsson I, Lamont RF. An update on the controversies of tocolytic therapy for the prevention of preterm birth. Acta Obstet Gynecol Scand 2003;82:1-9.

6. Sheenan A, Jones G, Hawken J, Crawshaw S, Judah $J$, et al. Fetal fibronectin test predicts delivery before 30 weeks of gestation in high risk women, but increases anxiety. BJOG 2005;112:293-298.

7. Lockwood CJ. Predicting premature delivery - no easy task. N Engl J Med 2002;346:282-4.

8. Rozenberg P, Goffinet F, Hessabi M. Comparison of the Bishop score, ultrasonographically measured cervical length, and fetal fibronectin assay in predicting time until delivery and type of delivery at term. Am J Obstet Gynecol 2000;182:108-13.

9. Hincz P, Wilczynski J, Kozarzewski M, Szaflik K. Twostep test: the combined use of fetal fibronectin and sonographic examination of the uterine cervix for prediction of preterm delivery in symptomatic patients. Acta Obstet Gynecol Scand 2002;81:58-63.

10. Gómez R, Romero R, Medina L, Nien JK, Chaiworapongsa $\mathrm{T}$, et al. Cervico vaginal fibronectin improves the prediction of preterm delivery based on sonographic cervical length in patients with preterm uterine contractions and intact membranes. Am J Obstet Gynecol 2001;184:652-5.

11. Plaut MM, Smith W, Kennedy K. Fetal fibronectina: The impact of a rapid test on the treatment of women with preterm labor symptoms. Am J Obstet Gynecol 2003;188:1588-93.

12. Tekesin I, Marek S, Hellmeyer L, Reitz D, Schmidt S. Assessment of rapid fetal fibronectin in predicting preterm delivery. Obstest Gynecol 2005;105:280-4. 